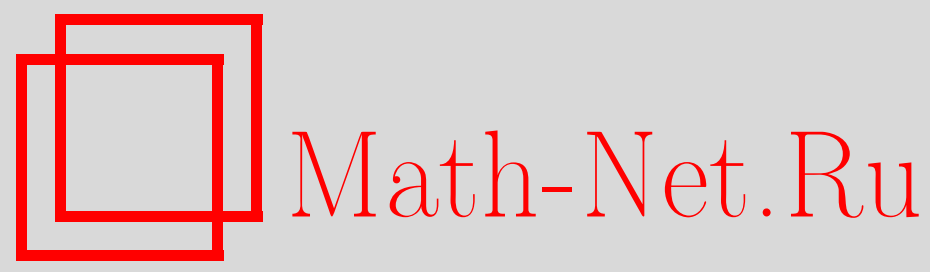

А. В. Карасев, Бесконечномерное 4-многообразие конечной когомологической размерности при СН, Матем. заметки, 1999, том 66, выпуск 5, 664-670

DOI: https://doi.org/10.4213/mzm1210

Использование Общероссийского математического портала Math-Net.Ru подразумевает, что вы прочитали и согласны с пользовательским соглашением http://www . mathnet.ru/rus/agreement

Параметры загрузки:

IP : 54.198 .187 .58

26 апреля 2023 г., $14: 13: 30$

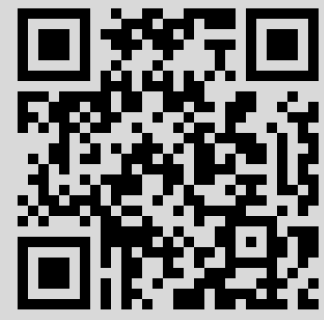




\section{БЕСКОНЕЧНОМЕРНОЕ 4-МНОГООБРАЗИЕ КОНЕЧНОЙ КОГОМОЛОГИЧЕСКОЙ РАЗМЕРНОСТИ ПРИ СН}

\section{А. В. Карасев}

В предположении континиуум-гипотезы построено дифференцируемое 4-многообразие $M$ размерности $\operatorname{dim} M=\infty$ и когомологической размерности сA $-\operatorname{dim} M=4$. Пространство $M$ совершенно нормально и наследственно сепарабельно.

Библиограффия: 9 названий.

1. Введение. В работе [1] В. В. Федорчуком в предположении континуум-гипотезы $(\mathrm{CH})$ для каждых $n, m$, удовлетворяющих неравенству $4 \leqslant n<m$, было построено дифференцируемое, совершенно нормальное, наследственно сепарабельное $n$-многообразие $M^{n, m}$ размерности $m-1 \leqslant \operatorname{dim} M^{n, m} \leqslant m \leqslant m+n-3 \leqslant \operatorname{Ind} M^{n, m} \leqslant$

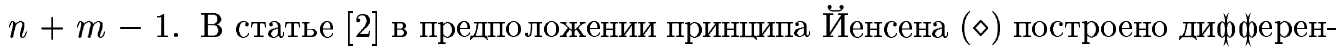
цируемое, счетно компактное, совершенно нормальное, наследственно сепарабельное $n$-многообразие $M^{n, m}$ размерности

$$
m=\mathrm{cA}-\operatorname{dim} M^{n, m}<\operatorname{dim} M^{n, m}=\infty,
$$

и был поставлен следующий вопрос: можно ли построить многообразие $M^{n}$, удовлетворяющее условию (1) в предположении континуум-гипотезы $(\mathrm{CH})$. В частности, можно ли, используя конструкцию примера [1] с применением идей, изложенных в [2], построить пример такого многообразия.

Результатом данной работы является положительный ответ на поставленньй вопрос, а именно:

Теорема. В предположении СН существует дифференцируемое, совериенно нормальное, наследственно сепарабельное 4-многообразие $M$ размерности $4=\mathrm{cA}-\operatorname{dim} M<\operatorname{dim} M=\infty$.

2. Предварительные сведения. Напомним необходимые сведения, которые понадобятся нам в дальнейшем.

ПРЕДЛОЖЕНИЕ 1 (см. [1, предложение 1.5]). Пусть $f: X \rightarrow Y$-монотонное, замкнутое отображсние на $Y, A$ и $B$ - замкнутые дизбюнктные подмножсества $Y$. Тогда для любой перегородки $C$ в пространстве $X$ между множсествами $f^{-1} A$ $u f^{-1} B$ мнохсество $f(C)$ является перегородкой в пространстве $Y$ мехду множествами $A$ и $B$.

Работа выполнена при поддержке Российского фонда фундаментальных исследований, грант № 97-01-00357. 
Теорема 1 (см. [3, гл. 5, теорема 15]). Пусть $X$ - нормальное пространство. Тогда $\operatorname{dim} X \geqslant n \Leftrightarrow$ существуют пары $\left(A_{i}, B_{i}\right), \quad 1 \leqslant i \leqslant n$, непересекающихся замкнутых подмножеств пространства $X$ такие, что $\cap\left\{C_{i}: 1 \leqslant i \leqslant n\right\} \neq \varnothing$ дя любых перегородок $C_{i}$ в пространстве $X$ межсду множсествами $A_{i} u B_{i}$.

СлЕДСТВИЕ 1. Если $\operatorname{dim} X \geqslant n$ для нормального пространства $X$, то существуют такие пары $\left(A_{i}, B_{i}\right), 1 \leqslant i \leqslant n-1$, непересекающ,хся замкнутых подмножеств пространства $X$, что $\operatorname{dim} \cap\left\{C_{i}: 1 \leqslant i \leqslant n-1\right\} \geqslant 1$ для любых перегородок $C_{i}$ в пространстве $X$ между множествами $A_{i} u B_{i}$.

ОПРЕДЕЛЕНИЕ 1 (см. [4]). Когомологической размерностью сА- $\operatorname{dim} X$ нормального пространства $X$ называется наибольшее целое число $n$, для которого найдется такое замкнутое в $X$ множество $F$, что спектральная группа целочисленных когомологий $H^{n}(X, F ; G)$, основанная на системе всех конечных открытых покрытий, отлична от нуля.

ОПРЕДЕЛЕНИЕ 2 (см. [5]). Когомологической размерностью с- $\operatorname{dim}_{G} X$ локально бикомпактного пространства $X$ относительно абелевой группы коэффициентов $G$ назьвается наибольшее целое число $n$, для которого существует такое бикомпактное множество $A \subset X$, что $H^{n}(A, G) \neq 0$, где $H^{i}(Y, G)-i$-я групша когомологий локально бикомпактного пространства $Y$ с бикомпактными носителями.

Будем обозначать группу $H^{i}(Y, Z)$ через $H^{i}(Y), \mathrm{c}-\operatorname{dim}_{Z} X$ через с - $\operatorname{dim} X$, где $Z-$ группа целых чисел. Число с $-\operatorname{dim} X$ будем назьвать когомологической размерностью локально бикомпактного пространства $X$.

Теорема 2 (см. [6]). Если локально бикомпактное простаранство X представлено в виде счетной суммы своих локально бикомпактных подмножеств $X_{k}$, $k \in \omega, \operatorname{moc}-\operatorname{dim} X=\sup \left\{\mathrm{c}-\operatorname{dim} X_{k}: k \in \omega\right\}$.

Теорема 3 (см. [4]). Для произвольного нормального пространства $X$ имеем $\mathrm{cA}-\operatorname{dim} X=\mathrm{cA}-\operatorname{dim} \beta X=\mathrm{c}-\operatorname{dim} \beta X$.

СлЕдСтвиЕ 2. Если $X-$ бикомпакт, mo $\mathrm{AA}-\operatorname{dim} X=\mathrm{c}-\operatorname{dim} X$.

Заметим, что для $n$-многообразия $M$ вьполняется равенство с $-\operatorname{dim} M=n$ (см. [2, равенство 4.3]).

Теорема 4 (см. [6, теорема 16]). Пусть $X$ наследственно паракомпактно. Тогда $\mathrm{cA}-\operatorname{dim} Y \leqslant \mathrm{cA}-\operatorname{dim} X$ для произвольного подмножества $Y$ в $X$.

3. Пример. Теперь построим многообразие $M$. Мы будем использовать конструкцю, изложенную в [1], модифицировав ее исходя из идей примера в [2].

Пусть $M_{1}$ - универсальная менгеровская кривая, $f: M_{1} \rightarrow Q$-непрерьвное отображение $M_{1}$ на гильбертов куб $Q$, все слои которого гомеоморфны $M_{1}$ (см. [5], [7], [8]).

Так как $M_{1}$ - одномерньй континуум, существует вложение $M_{1}$ в трехмерную сферу $S^{3}$. Фиксируем такое вложение $M_{1} \subset S^{3}$, сферу $S^{3}$ считаем границей замкнутого шара $B^{4}$. Пусть $\mathcal{D}$ - бесконечномерньй компакт когомологической размерности с $\operatorname{dim} \mathcal{D}=3$, построенньй А.Н. Дранишниковым [9]. Фиксируем вложение $\mathcal{D}$ в $Q$. Пусть $A=f^{-1} \mathcal{D}$. Через $\mathcal{F}_{\mathcal{D}}$ обозначим следующее разбиение шара $B^{4}: \mathcal{F}_{\mathcal{D}}=\left\{f^{-1}(q)\right.$ : $q \in \mathcal{D}\} \cup\left\{\{x\}: x \in B^{4} \backslash A\right\}$. Фактор-отображение $B^{4} \rightarrow B^{4} / \mathcal{F}_{\mathcal{D}}$ обозначим через $\varphi$, 
фактор-пространство $B^{4} / \mathcal{F}_{\mathcal{D}}$ через $B_{\varphi}^{4}$. Положим $S_{\varphi}^{3}=\varphi\left(S^{3}\right)$. Заметим, что $\mathcal{D}=f(A)$ естественно гомеоморфно $\varphi(A)$ и, следовательно, вложено в $S_{\varphi}^{3}$.

Точку $y \in S_{\varphi}^{3}$ назовем точкой первого рода, если множество $\varphi^{-1}(y)$ состоит из одной точки, назовем точку у точкой второго рода, если $\varphi^{-1}(y)$ совпадает с одним из континуумов $f^{-1}(q), q \in \mathcal{D}$.

Пусть $\left\{h_{\alpha}: \alpha<\omega_{1}\right\}$ - какая-нибудь нумерация точек множества $S_{\varphi}^{3}$, существующая в силу континуум-гипотезы.

Теперь совершенно аналогично [1] построим обратньй спектр $S=\left\{X_{\beta}, \pi_{\gamma}^{\beta}: \gamma \leqslant \beta<\right.$ $\left.\omega_{1}\right\}$, удовлетворяющий следующим свойствам (см. [1]).

1. $X_{\beta}$ гомеоморфно $B_{\varphi}^{4}$ для любого $\beta<\omega_{1}$.

2. Существует $Z_{\beta} \subset X_{\beta}$ такое, что $Z_{\beta}$-гладкое 4 -многообразие с дифференциальной структурой $\mathcal{D}_{\beta}$, причем $X_{\beta} \backslash Z_{\beta}$ гомеоморфно $S_{\varphi}^{4}$.

3. $\pi_{\gamma}^{\beta}$ - почти гомеоморфизм при $\gamma<\beta$.

4. Если $\gamma<\omega$, то $\left(\pi_{\gamma}^{\gamma+1}\right)^{-1} h_{\gamma}=h_{\gamma} \cup J_{\gamma+1} \equiv K_{\gamma+1}$ - гомеоморфно отрезку $I$, когда $h_{\gamma}$ есть точка первого рода, и конусу над произведением $M_{1} \times I$, когда $h_{\gamma}$ - точка второго рода.

5. $\left.\pi_{\gamma}^{\gamma+1}\right|_{\left(\pi_{\gamma}^{\gamma+1}\right)^{-1}\left(Z_{\gamma}\right)}$ - гомеоморфизм.

6. Если $\delta<\gamma<\beta$ и $h_{\gamma}$ - предельная точка множества $\pi_{0}^{\beta}\left(A_{\delta}\right)$, то всякая точка $x \in K_{\gamma+1}$ является предельной точкой множества счетных подмножеств множества $Z=$ $\cup\left\{Z_{\alpha}: \alpha<\omega_{1}\right\}$ таких, что каждое счетное подмножество множества $Z$ занумеровано несчетным множеством индексов (эта нумерация существует в силу $\mathrm{CH}$ ).

7. $\left(Z_{\beta}, \mathcal{D}_{\beta}\right)$ диффеоморфно $\mathbb{R}^{4}$, т.е. атлас $\mathcal{D}_{\beta}$ содержит такую карту $\left(Z_{\beta}, \varphi_{\beta}\right)$, что $\varphi_{\beta}\left(Z_{\beta}\right)=\mathbb{R}^{4}$.

8. Если $\gamma<\beta$, то $\left(Z_{\gamma}, \varphi_{\gamma}\right) \in \mathcal{D}_{\beta}$.

9. $\left.\pi_{\alpha}^{\beta}\right|_{\left(S_{\varphi}^{3}\right)}-$ тождественно при любых $\alpha \leqslant \beta<\omega_{1}$.

Пусть $X$ - предел обратного спектра $S$. Положим $M=(Z, \mathcal{D})$, где $Z=\cup Z_{\alpha}, \mathcal{D}=$ $\cup\left\{\mathcal{D}_{\beta}: \beta<\omega_{1}\right\}$. Тогда $X-$ бикомпакт, а дифференцируемое 4-многообразие $M$ является его открытьм всюду плотным подмножеством, причем разность $X \backslash M$ гомеоморфна компакту $S_{\varphi}^{3}$.

Из свойства 9 спектра $S$ следует, что

$$
\left.\pi_{\alpha}\right|_{S_{\varphi}^{3}} \quad \text { - тождественное отображение. }
$$

4. Свойства многообразия $M$. Пусть $\pi_{\alpha}: X \rightarrow X_{\alpha} \equiv B_{\varphi}^{4}-$ сквозная проекция спектра $S$.

Тогда верно следующее

ПРЕДЛОЖЕНИЕ 2. 1. Если $x \in X_{\alpha} \backslash S_{\varphi}^{3}$, mo $\pi_{\alpha}^{-1} x$ coстоит из одной точки.

2. Если $h \in S_{\varphi}^{3}-$ точка первого рода, то множество $\pi_{\alpha}^{-1} h$ гомеоморофно отрезку $I$.

3. Если $h \in S_{\varphi}^{3}-$ точка второго рода, то $\pi_{\alpha}^{-1} h$ гомеоморфно конусу над произведением $M_{1} \times I$.

В статье [1] приведено доказательство этого предложения в случае, когда $\alpha=0$, однако нетрудно видеть, что это доказательство дословно переносится на случай произвольного $\alpha<\omega_{1}$.

Положим $p_{\alpha}=\left.\pi_{\alpha}\right|_{M}$. 
ПРЕДЛОЖЕНИЕ 3 (Следствие из п. 1 в предложении 2). Имеем $p_{\alpha} \mid p_{\alpha}^{-1}\left(X_{\alpha} \backslash S_{\varphi}^{3}\right)=$ $p_{\alpha}^{-1}\left(Z_{\alpha}\right)$ - гомеоморфизм.

Пусть $f: X \rightarrow Y$ - отображение; тогда для $A \subset X$ положим $f^{\#} A=\{y \in Y$ : $\left.f^{-1} y \subset A\right\}=Y \backslash f(X \backslash A)$.

ПРЕДЛОЖЕНИЕ 4 (Основное свойство отображения $p_{\alpha}$ ). Для всякого замкнутого множества $F \subset M$ и открытого множества $U \subset M$ множества $\overline{p_{\alpha}(F)_{\alpha}} \backslash p_{\alpha}^{\#}(F)$ и $p_{\alpha}(U) \backslash \operatorname{Int} p \alpha \# U$ не более чем счетны для любого $\alpha<\omega_{1}$.

(Доказательство для $\alpha=0$ легко обобщается на случай произвольного $\alpha<\omega_{1}$, cм. [1].)

Для открытого множества $U \subset M$ положим $\tilde{U}=X \backslash \overline{(M \backslash U)} X$. Тогда из основного свойства отображения $p_{\alpha}$ вытекает

СлЕДСТВИЕ 3. Множество $\pi_{\alpha}(\widetilde{U}) \backslash \pi_{\alpha}^{\#}(\tilde{U})$ счетно.

ПРЕДЛОЖЕНИЕ 5 (см. предложение 2.2 в [1]). Многообразие $M$ совершенно нормально и наследственно сепарабельно.

\section{5. Размерностные свойства многообразия $M$.}

ПРЕДЛОЖЕНИЕ 6. Имеет место равенство $\operatorname{dim} M=\infty$.

ДокАЗАТЕльство. Нам достаточно показать, что $\operatorname{dim} M \geqslant n$ для любого $n \in \mathbb{N}$.

Заметим, что компакт Дранишникова $\mathcal{D}$ вложен в $S_{\varphi}^{3}$ (см. раздел 3 ). Следовательно, $\operatorname{dim} S_{\varphi}^{3}=\infty$, поэтому, так как $S_{\varphi}^{3} \subset B_{\varphi}^{4}, \operatorname{dim} B_{\varphi}^{4}=\infty$.

Поэтому согласно следствию 1 для любого $n \in \mathbb{N}$ найдутся $\left(A_{i}^{1}, A_{i}^{2}\right), 1 \leqslant i \leqslant n+1,-$ такие пары дизъюнктных замкнутых подмножеств компакта $X_{0}=B_{\varphi}^{4}$, что

$$
\operatorname{dim} \cap\left\{B_{i}: 1 \leqslant i \leqslant n\right\} \geqslant 1
$$

для любых перегородок $B_{i}$ между $A_{i}^{1}$ и $A_{i}^{2}$.

Пусть $U_{i}^{j}, j=1,2,-$ такие окрестности множеств $A_{i}^{j}$, что $\bar{U}_{i}^{1} \cap \bar{U}_{i}^{2}=\varnothing$ для всех $i$, и пусть $F_{i}^{j}=M^{4} \cap \pi_{0}^{-1} \bar{U}_{i}^{j}=p_{0}^{-1} \bar{U}_{i}^{j}$.

Согласно теореме 1 для проверки неравенства $\operatorname{dim} M \geqslant n$ нам достаточно показать, что $\cap\left\{C_{i}: 1 \leqslant i \leqslant n\right\} \neq \varnothing$ для любых перегородок $C_{i}$ между множествами $F_{i}^{1}$ и $F_{i}^{2}$.

Пусть $M \backslash C_{i}=V_{i}^{1} \cup V_{i}^{2}$, где $V_{i}^{1}$ и $V_{i}^{2}$ являются дизъюнктными окрестностями множеств $F_{i}^{1}$ и $F_{i}^{2}$. Положим $\mathcal{D}_{i}=X \backslash\left(\tilde{V}_{i}^{1} \cup \widetilde{V}_{i}^{2}\right)$. Так как $\pi_{0}^{-1} U_{i}^{j} \subset \widetilde{V}_{i}^{j}$, множество $\mathcal{D}_{i}$ является перегородкой в бикомпакте $X$ между $\pi_{0}^{-1} A_{i}^{1}$ и $\pi_{0}^{-1} A_{i}^{2}$.

Заметим, что отображение $\pi_{0}$ удовлетворяет условию предложения 1 в силу бикомпактности $X$ и $X_{0}$, а также свойствам 1-3 проекций $\pi_{\alpha}$ (см. предложение 2 ). Поэтому $\pi_{0} \mathcal{D}_{i}$ - перегородка в $X_{0}$ между $A_{i}^{1}$ и $A_{i}^{2} ;$ следовательно, согласно $(3) \operatorname{dim} \cap\left\{\pi_{0}\left(\mathcal{D}_{i}\right):\right.$ $1 \leqslant i \leqslant n\} \geqslant 1$.

Таким образом, нам достаточно показать что множество $\cap_{i} \pi_{0}\left(\mathcal{D}_{i}\right) \backslash \bigcap_{i} P_{0}^{\#} C_{i}$ счетно. Отсюда будет вытекать, что $\cap\left\{C_{i}: 1 \leqslant i \leqslant n\right\} \neq \varnothing$.

Легко проверить следуюшие теоретико-множественные включения:

$$
\begin{aligned}
& \bigcap_{\alpha} P_{\alpha} \backslash \bigcap_{\alpha} Q_{\alpha} \subset \bigcup_{\alpha}\left(P_{\alpha} \backslash Q_{\alpha}\right), \\
& \bigcup_{\alpha} P_{\alpha} \backslash \bigcup_{\alpha} Q_{\alpha} \subset \bigcup_{\alpha}\left(P_{\alpha} \backslash Q_{\alpha}\right) .
\end{aligned}
$$


Поэтому нам достаточно показать, что $\pi_{0}\left(\mathcal{D}_{i}\right) \backslash P_{0}^{\#} C_{i}$ счетно, а для этого согласно основному свойству отображения $p_{\alpha}$ достаточно показать, что $\pi_{0}\left(\mathcal{D}_{i}\right) \backslash p_{0}\left(C_{i}\right)$ счетно. Имеем: $X_{0} \backslash p_{0}\left(C_{i}\right) \subset p_{0}\left(V_{i}^{1}\right) \cup p_{0}\left(V_{i}^{2}\right)$ и $\pi_{0}\left(\mathcal{D}_{i}\right) \subset X_{0} \backslash p_{0}^{\#} \widetilde{V}_{i}^{1} \cup p_{0}^{\#} \widetilde{V}_{i}^{2}$. Следовательно, $\pi_{0}\left(\mathcal{D}_{i}\right) \backslash p_{0}\left(C_{i}\right) \subset p_{0}\left(V_{i}^{1}\right) \cup p_{0}\left(V_{i}^{2}\right) \backslash \pi_{0}^{\#} \widetilde{V}_{i}^{1} \cup \pi_{0}^{\#} \tilde{V}_{i}^{2} \cup \widetilde{V}_{i}^{2} \underset{(5)}{\subset}\left(p_{0}\left(V_{i}^{1}\right) \backslash \pi_{0}^{\#} \widetilde{V}_{i}^{1}\right) \cup$ $\left(p_{0}\left(V_{i}^{2}\right) \backslash \pi_{0}^{\#}\left(U_{i}^{2}\right)\right) \subset\left(\pi_{0}\left(\tilde{V}_{i}^{1}\right) \backslash \pi_{0}^{\#} V_{i}^{1}\right) \cup\left(\pi_{0}\left(\tilde{V}_{i}^{2}\right) \backslash \pi_{0}^{\#}\left(\tilde{V}_{i}^{2}\right)\right)$.

Но последнее множество счетно в силу следствия из основного свойства отображений $p_{\alpha}$.

Итак, $\operatorname{dim} M \geqslant n$ для всех $n \in \mathbb{N} ;$ следовательно, $\operatorname{dim} M=\infty$.

ПРЕДЛОЖЕНИЕ 7. Имеем $\mathrm{cA}-\operatorname{dim} B_{\varphi}^{4}=4$.

ДокАЗАТЕЛЬСТво. В силу следствия 2 и теоремы $2 \mathrm{cA}-\operatorname{dim} B_{\varphi}^{4}=c-\operatorname{dim} B_{\varphi}^{4}=$ $\sup \left\{c-\operatorname{dim} \mathcal{D}, c-\operatorname{dim} B_{\varphi}^{4} \backslash \mathcal{D}\right\}=\max \{3,4\}=4$. Предложение доказано.

Пусть $X$ - нормальное пространство, $F$ - его замкнутое подмножество, $u=\left\{U_{i}\right.$ : $1 \leqslant i \leqslant k\}$ - открытое покрытие $X$. Тогда через $K_{u}$ мы будем обозначать симплициальньй комплекс, являющийся нервом покрытия $u$, а через $K_{u, F}-$ подкомплекс в $K_{u}$, являющийся нервом покрытия $u$, ограниченного на множество $F$.

Если дано другое открытое покрытие $v=\left\{V_{i}: 1 \leqslant i \leqslant k\right\}$, которое комбинаторно вписано в $u$, т.е. $V_{i} \subseteq U_{i}$ для любого $i$, то через $\mu_{u, v}$ мы будем обозначать гомоморфизм группы когомологий $H^{n}\left(K_{u}, K_{u, F} ; G\right)$ в группу когомологий $H^{n}\left(K_{v}, K_{v, F} ; G\right)$, индуцированньй вписанностью покрытия $v$ в покрытие $u$.

ПРЕДЛОЖЕНИЕ 8. Имеем сА $-\operatorname{dim} M=4$.

Для доказательства этого предложения нам потребуется следующая

Лемма 1. Для любого открытого подмножества $U$ в $M$ найдется $\alpha<\omega_{1}$ такое, что для всех $\beta \geqslant \alpha$ выполняется равенство $p_{\beta}^{\#}(U)=p_{\beta}(U)$, причем $p_{\beta}(U)$ открыто в $X_{\beta}$.

ДокАЗАТЕЛЬСТво. Согласно основному свойству отображения $p_{0}$ разность $p_{0} U \backslash \operatorname{Int} p_{0}^{\#} U-$ не более чем счетна. Нетрудно видеть, что $p_{0}(U) \backslash \operatorname{Int} p_{0}^{\#} U \subset S_{\varphi}^{3}$. Пусть $p_{0}(U) \backslash \operatorname{Int} p_{0}^{\#} U=\left\{h_{\alpha_{i}}: i<\omega\right\}$. Положим $\alpha=\sup \left\{\alpha_{i}\right\}$ и проверим, что $\alpha$ удовлетворяет требуемому свойству. Покажем сначала, что $p_{\alpha}^{\#} U=p_{\alpha} U$. Действительно, предположим, что существует $x \in p_{\alpha} U \backslash p_{\alpha}^{\#} U$. Тогда $\pi_{0}^{\alpha} x \in p_{0} U \backslash p_{0}^{\#} U \subseteq p_{0}^{\#} U \backslash \operatorname{Int} p_{0}^{\#} U$, т.е.

$$
\pi_{0}^{\alpha} x=h_{\alpha_{i}} \text { для некоторого } i .
$$

Так как $x \in p_{\alpha} U$, существует $y \in U$ такая, что $p_{\alpha} y=x$.

Согласно (6) $y \in p_{0}^{-1} h_{\alpha_{i}}$. Заметим, что $p_{0}^{-1}\left(h_{\alpha_{i}}\right)=p_{\alpha}^{-1}\left(\left(\pi_{0}^{\alpha}\right)^{-1} h_{\alpha_{i}}\right)$ и в силу свойств $3,5,9$ спектра $S$, а также определения многообразия $M$ отображение $\left.p_{\alpha}\right|_{p_{\alpha}^{-1}\left(\left(\pi_{0}^{\alpha}\right)^{-1} h_{\alpha_{i}}\right)}$ является гомеоморфизмом; следовательно, $x=p_{\alpha} y \in p_{\alpha}^{\#} U$, что противоречит выбору $x$.

Теперь мыпокажем, что $p_{\alpha} U$ открыто, а именно, $p_{\alpha} U=p_{\alpha}\left(U \cap Z_{\alpha}\right) \cup\left(\pi_{0}^{\alpha}\right)^{-1}\left(\operatorname{Int} p_{0}^{\#} U\right)$.

Заметим, что $p_{\alpha}\left(U \cap Z_{\alpha}\right)$ открыто, так как согласно определению многообразия $M$ $Z_{\alpha}$ является одной из открытых карт в атласе $M$ и $p_{\alpha} \mid z_{\alpha}$ - гомеоморфизм (см. определение многообразия $M$ и свойство 5 спектра $S$ ). Поэтому нам необходимо проверить включение

$$
p_{\alpha} U \subset p_{\alpha}\left(U \cap Z_{\alpha}\right) \cup\left(\pi_{0}^{\alpha}\right)^{-1}\left(\operatorname{Int} p_{0}^{\#} U\right)
$$


Обратное включение очевидно.

Пусть $x \in p_{\alpha} U$. Предположим, что $x \notin\left(\pi_{0}^{\alpha}\right)^{-1}\left(\operatorname{Int} p_{0}^{\#} U\right)$. Тогда $\pi_{0}^{\alpha} x=h_{\alpha_{i}}$ для некоторого $i$. В силу выбора $\alpha$, свойств $3,5,9$ спектра $S$ и определения многообразия $M$ $p_{\alpha}^{-1}(x) \subset p_{\alpha}^{-1}\left(\left(\pi_{0}^{\alpha}\right)^{-1} h_{\alpha_{i}}\right)=p_{\alpha}^{-1}\left(\left(\pi_{0}^{\alpha}\right)^{-1} h_{\alpha_{i}}\right) \backslash h_{\alpha_{i}} \subseteq p_{\alpha}^{-1}\left(Z_{\alpha}\right)=Z_{\alpha}$.

Таким образом, $p_{\alpha}^{-1} x \subseteq Z_{\alpha} \cap U$. Следовательно, $x \in p_{\alpha}\left(Z_{\alpha} \cap U\right)$. Включение (7) проверено. Очевидно, что требуемому условию удовлетворяет также и любое $\beta \geqslant \alpha$. Это завершает доказательство леммы.

Переходим к доказательству предложения 8.

В силу того, что $M$ является 4 -многообразием, $\mathrm{cA}-\operatorname{dim} M \geqslant 4$. Покажем, что сA $\operatorname{dim} M \leqslant 4$.

Пусть $u=\left\{U_{i}: 1 \leqslant i \leqslant k\right\}$ - произвольное конечное открытое покрытие многообразия $M, F$ - его произвольное замкнутое подмножество. Для доказательства неравенства $c A-\operatorname{dim} M \leqslant 4$ нам согласно определению 1 надо показать, что группы $H^{n}(M, F ; \mathbb{Z})$ тривиальны при $n>4$. В свою очередь, для этого согласно определению групшы $H^{n}(M, F ; \mathbb{Z})$ нам достаточно показать, что для любого элемента $z$ группы $H^{n}\left(K_{u}, K_{u, F} ; \mathbb{Z}\right)$ найдется такое покрытие $v=\left\{V_{i}: 1 \leqslant i \leqslant k\right\}$, комбинаторно вписанное в покрытие $u$, что образ элемента $z$ при гомоморфизме $\mu_{u v}$ когомологичен нулю, т.е. $\mu_{u v}(z)=0$ в группе $H^{n}\left(K_{v}, K_{v, F} ; \mathbb{Z}\right)$ при $n>4$.

Используя лемму 1 , для каждого $U_{i}$ найдем $\alpha_{i}<\omega_{1}$ такое, что $p_{\beta} U_{i}=p_{\beta}^{\#} U_{i}$ открыто в пространстве $X_{\beta}$ для каждого $\beta \geqslant \alpha_{i}$. Положим $\alpha^{\prime}=\max \left\{\alpha_{i}: 1 \leqslant i \leqslant k\right\}$.

Далее, согласно основному свойству отображения $p_{\alpha}$ разность $\overline{p_{\alpha^{\prime}} F} X_{\alpha^{\prime}} \backslash p_{\alpha^{\prime}}^{\#}$ не более

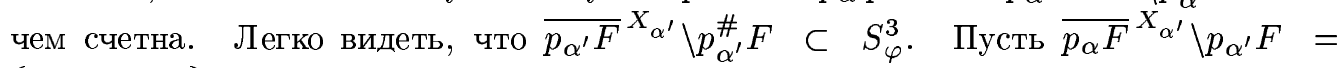
$\left\{h_{\beta_{i}}: i<\omega\right\}$.

Положим $\beta=\sup \beta_{i}, \alpha=\max \left\{\alpha^{\prime}, \beta\right\}, X_{\alpha}^{\prime}=\cup\left\{p_{\alpha} U_{i}: 1 \leqslant i \leqslant k\right\}, \Phi=p_{\alpha} F$, $O_{i}=p_{\alpha} U_{i}$.

Нетрудно видеть, что в силу выбора $\alpha$ пространство $X_{\alpha}^{\prime}$ и множество $\Phi$ обладают следуюшими свойствами:

1) $\Phi=p_{\alpha}^{\#} F, \Phi \subset X_{\alpha}^{\prime}$;

2) $\Phi$ замкнуто в пространстве $X_{\alpha}^{\prime}$;

3) $p_{\alpha}^{-1} X_{\alpha}^{\prime}=M$.

Положим $\omega=\left\{O_{i}: 1 \leqslant i \leqslant k\right\}$ - открытое покрытие $X_{\alpha}^{\prime}$. Тогда по построению множеств $O_{i}$ и $\Phi$ симплициальный комплекс $K_{u}$ совпадает с комплексом $K_{\omega}$, а подкомплекс $K_{u, F}$ совпадает с подкомплексом $K_{\omega, \Phi}$.

Согласно свойству 1 спектра $S X_{\alpha}$ гомеоморфно $B_{\varphi}^{4}$. Согласно предложению 7 сA $\operatorname{dim} X_{\alpha}=\mathrm{cA}-\operatorname{dim} B_{\varphi}^{4}=4$. Поскольку $B_{\varphi}^{4}-$ метризуемый компакт, мы находимся в условиях теоремы 4 , поэтому $\mathrm{cA}-\operatorname{dim} X_{\alpha}^{\prime}=4$. Следовательно, для любого элемента $z \in H^{n}\left(K_{\omega}, K_{\omega, \Phi} ; \mathbb{Z}\right)=H^{n}\left(K_{u}, K_{u, F} ; \mathbb{Z}\right)$ найдется покрытие $\tilde{v}=\left\{\widetilde{V}_{i}: 1 \leqslant\right.$ $i \leqslant k\}$ пространства $X_{\alpha}^{\prime}$, комбинаторно вписанное в $\omega$, такое, что $\mu_{\tilde{v} \omega}(z)=0$ в группе $H^{n}\left(K_{\tilde{v}}, K_{\tilde{v}, \Phi} ; \mathbb{Z}\right)($ где $n>4)$. Положим теперь $V_{i}=p_{\alpha}^{-1}\left(\widetilde{V}_{i}\right), v=\left\{V_{i}: 1 \leqslant i \leqslant k\right\}$. Тогда $v$ является конечным открытьм покрытием многообразия $M$, комбинаторно вписанным в $U$, причем комплекс $K_{v}$ совпадает с комплексом $K_{\tilde{v}}$, подкомплекс $K_{v, F}-$ с подкомплексом $K_{\tilde{v}, \Phi}$ и гомоморфизм $\mu_{\tilde{v} \omega}-$ с гомоморфизмом $\mu_{v u}$. Следовательно, $\mu_{v u}(z)=0$ в группе $H^{n}\left(K_{v}, K_{v, F} ; \mathbb{Z}\right)$.

Предложение 8 доказано.

Утверждение теоремы теперь следует из предложений 5, 6 и 8. 
Автор выражает признательность В. В. Федорчуку за постановку задачи и помощь в работе.

\section{СПИСОК ЦИТИРОВАННОЙ ЛИТЕРАТУРЫ}

[1] Федорчук В.В.Дифференцируемое многообразие с несовпадающими размерностями при СН // Матем. сб. 1995. Т. 186. № 1. С. 149-160.

[2] Федорчук В.В. О трансфинитной и когомологической размерностях 4-многообразий // Тр. МИАН. 1996. Т. 212. С. 193-212.

[3] Александров П. С., Пасынков Б. А. Введение в теорию размерности. М.: Наука, 1973.

[4] Alexandroff P.S. On the dimension of normal spaces // Proc. Roy. Soc. London. A. 1947. V. 189. P. 11-39.

[5] Anderson R. D. Monotone interior dimension-raising mappings // Duke Math. J. 1952. V. 19. P. 359-366.

[6] Кузьминов В. И. Гомологическая теория размерности // УМН. 1968. Т. 23. №5 (142).

[7] Anderson R. D. A continuous curve admitting monotone open maps onto all locally connnected metric continua // Bull. Amer. Math. Soc. 1956. V. 62. P. 265.

[8] Wilson D. C. Open mappings of the universal curve onto continuous curves // Trans. Amer. Math. Soc. 1972. V. 168. P. 497-515.

[9] Дранишников А. Н. О проблеме П. С. Александрова // Матем. сб. 1988. Т. 135. С. 551-557.

Московский государственный университет им. М.В. Ломоносова

Поступило

E-mail: akarasev@academy.ru 22.06 .1998 